\title{
Qishen Granule Improved Cardiac Remodeling via Balancing M1 and M2 Macrophages
}

\section{OPEN ACCESS}

Edited by:

George Qian Li,

Western Sydney University,

Australia

Reviewed by:

Geng Wenye,

Fudan University, China

Yi Ding,

Fourth Military Medical University,

China

${ }^{*}$ Correspondence:

Yue Liu

liuyueheart@hotmail.com

Yong Wang

doctor_wangyong@163.com

Chun Li

lichun19850204@163.com

tThese authors have contributed equally to this work

Specialty section: This article was submitted to

Ethnopharmacology,

a section of the journal

Frontiers in Pharmacology

Received: 03 July 2019 Accepted: 01 November 2019 Published: 25 November 2019

Citation:

LuW, Wang $Q$, Sun $X, \mathrm{He} H$, Wang $Q$, Wu Y, Liu Y, Wang Y and Li C (2019) Qishen Granule Improved Cardiac Remodeling via Balancing

M1 and M2 Macrophages.

Front. Pharmacol. 10:1399. doi: 10.3389/fphar.2019.01399

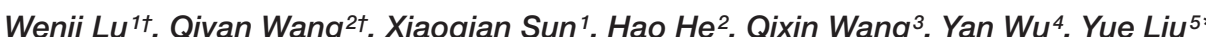
Yong Wang ${ }^{1 *}$ and Chun $\mathrm{Li}^{3 *}$

${ }^{1}$ College of Chinese Medicine, Beijing University of Chinese Medicine, Beijing, China, ${ }^{2}$ School of Life Sciences, Beijing University of Chinese Medicine, Beijing, China, ${ }^{3}$ Modern Research Center for Traditional Chinese Medicine, School of Chinese Materia Medica, Beijing University of Chinese Medicine, Beijing, China, ${ }^{4}$ Center of Scientific Experiment, Beijing University of Chinese Medicine, Beijing, China, ${ }^{5}$ Cardiovascular Disease Center, Xiyuan Hospital, China Academy of Chinese Medical Sciences, Beijing, China

Macrophages play a pivotal role in myocardial remodeling (MR) process which could eventually lead to heart failure. Splenic monocytes could be mobilized and recruited under inflammatory conditions and differentiated into different types of macrophages in heart tissues. Inflammatory M1 macrophages could aggravate tissue damage whereas M2 macrophages could promote angiogenesis and tissue repair process. Unbalanced ratio of M1/M2 macrophages may eventually lead to adverse remodeling. Therefore, regulating differentiation and activities of macrophages are potential strategies for the management of myocardial remodeling. Qishen Granule (QSG) is an effective Chinese medicine for treating heart failure. Our previous studies demonstrated that QSG could inhibit myocardial fibrosis through regulating secretion of cytokines and activation of macrophages. However, the detailed effects of QSG on had not been elucidated yet. In this study, we aimed to explore the effect of QSG on the release of splenic monocytes, the recruitment of monocytes into heart tissues and the differentiation of macrophages under ischemic conditions. Our results showed that QSG could suppress the release of monocytes from the spleen and recruitment of monocytes to heart tissues via inhibiting splenic angiotensin (Ang) II/AT1-cardiac monocyte chemotactic protein (MCP)-1/CC chemokine receptor 2 (CCR2) pathway. The anti-fibrotic effect of QSG was exerted by inhibiting M1 macrophage-activated transforming growth factor (TGF)- $\beta 1 / \mathrm{Smad} 3$ pathway. Meanwhile, QSG could promote angiogenesis by promoting differentiation of M1 macrophages into M2 macrophages. Our results suggest that compounds of Chinese medicine have synergistic effects on cardiac and splenic organs through regulating differentiation of monocytes/macrophages in inhibiting myocardial remodeling.

Keywords: Qishen Granule, myocardial fibrosis, splenic monocytes, macrophages, angiogenesis 


\section{INTRODUCTION}

Myocardial remodeling (MR) is an important pathophysiological change after myocardial infarction, and is a common pathological change during the development of heart failure (HF) (Pryds et al., 2019). Macrophage-mediated inflammation contributes greatly to fibrosis and prolonged inflammation will eventually lead to MR. Macrophages also participate in the healing process after myocardial infarction. Therefore, regulating differentiation and activities of macrophages are potential strategies for the management of MR to prevent HF (Honold and Nahrendorf, 2018).

The myocardium is mainly composed of cardiomyocytes, fibroblasts, endothelial cells and macrophages ( $\mathrm{Hu}$ et al., 2017). Macrophages are involved in myocardial injury, repair and remodeling processes during the inflammatory response (Hulsmans et al., 2018). In steady state, most cardiac macrophages are derived from local progenitors and their functions remain largely unknown. It is speculated that these resident macrophages may play roles in guarding against infection, regulating angiogenesis and directing matrix turnover (Jablonski et al., 2015). Under inflammatory condition, such as myocardial infarction, circulating monocytes will be abundantly recruited to ischemic heart tissues through monocyte chemotactic protein-1 (MCP-1)/CC chemokine receptor 2 (CCR2) interactions. Once recruited, monocytes can differentiate into different types of macrophages which participate either in inflammatory or repairing process. According to surface markers and functions, macrophages can be roughly classified into inflammatory M1 and less inflammatory M2 types. M1 macrophages participate in inflammatory response by secreting pro-inflammatory cytokines interleukin (IL)-6 and chemokines, which aggravate tissue damage (Momtazi-Borojeni et al., 2019). M2 macrophages have only weak antigen-presenting ability, and they secrete vascular endothelial growth factor (VEGF) which promotes angiogenesis and tissue repair (Wu et al., 2010). The dynamic regulation and balance between M1 and M2 macrophages will determine the ventricular remodeling process.

The spleen is the largest organ in the immune system. Besides, it is a huge reservoir of more than half of the body's monocytes (Halade et al., 2018). About $40-70 \%$ of monocytes that are recruited to myocardial infarct site are derived from the spleen (Sager et al., 2017). The resting spleen contains a large reservoir of bona fide monocytes (Robbins and Swirski, 2010). Under inflammatory condition, angiotensin (Ang) II activates the Ang II type I receptor (AT1) in the spleen, allowing a large number of monocytes to be released into the blood. The role that spleen plays in myocardial infarction remains to be fully investigated.

Abbreviations: Ang II, angiotensin II; CCR2, CC chemokine receptor 2; EF, ejection fraction; FS, fractional shortening; HF, heart failure; IL-6, Interleukin-6; LAD, left anterior descending; LVAW;s, left ventricular anterior wall; systole; LVAW;d, left ventricular anterior wall; diastole; LVID;d, left ventricular internal diameter; diastole; LVID;s, left ventricular internal diameter; systole; LVPW;s, left ventricular posterior wall; systole; LVPW; d, left ventricular posterior wall; diastole; MR, myocardial remodeling; MCP-1, monocyte chemotactic protein-1; MMP2, Matrix metallopeptidase 2; QSG, Qishen Granule; RAAS, renin angiotensin aldosterone system; TGF- $\beta 1$, transforming growth factor- $\beta 1$; VEGF, vascular endothelial growth factor.
Inhibiting release of monocytes from spleen could reduce the number of macrophages in heart tissues and ameliorate inflammation (Partha D. Nahrendorf, 2015). Therefore, spleen is a potential target in the management of MR.

Qishen Granule (QSG) is prepared from the combination of the classic prescription Zhenwu Tang and Si Miao Yong An Tang in traditional Chinese medicine (Li et al., 2014). QSG mainly consists of six Chinese herbs including A. membranaceus (Fisch.) Bunge., S. Miltiorrhiza Bunge., L. japonica Thunb., S. aestivalis Griseb., A. fischeri Rchb. and G. uralensis Fisch (Li et al., 2016). The chemical component of QSG has been verified by liquid chromatography-mass spectrometry techniques (Guo et al., 2016). Preclinical studies have proven that QSG can effectively improve cardiac function and inhibit myocardial fibrosis (Wang et al., 2019). It has also been reported that QSG could protect against myocardial damage after macrophage activation (Liu et al., 2016). However, it hasn't been clarified if QSG could inhibit activation of macrophages through inhibiting release of monocytes from spleen. The regulative effect of QSG on differentiation of macrophages is also unclear. In this study, we aimed to explore if QSG could ameliorate myocardial remodeling through suppressing release of monocytes from spleen and inhibiting macrophage recruitment in a HF rat model. The roles of QSG on macrophage differentiation and splenic AngII/AT1cardiac MCP-1/CCR2 pathway were investigated. This study will provide alternative targets in the management of macrophageinduced myocardial remodeling.

\section{MATERIALS AND METHODS}

\section{Animals}

All animal experimental protocols were approved by the Ethics Committee of Beijing University of Chinese Medicine and conformed to the Guide for the Care and Use of Laboratory Animals published by the U.S. National Institute of Health (NIH Publication No. 85-23, revised 1996). 60 male Sprague-Dawley (SD) healthy rats $(220 \pm 20 \mathrm{~g})$ in Specific Pathogen Free (SPF) grade used in this study were obtained from Beijing Vital River Laboratory Animal Technology Co. Ltd (China). The rats were housed in the cages under standard laboratory conditions $(12 \mathrm{~h}$ light/dark cycle, controlled temperature of $25^{\circ} \mathrm{C}$ ).

\section{Drugs}

Qishen Qranule is composed of A. membranaceus (Fisch.) Bunge., S. Miltiorrhiza Bunge., L. japonica Thunb., S. aestivalis Griseb., A. fischeri Rchb. and G. uralensis Fisch, purchased from Beijing Tongrentang (Group) Co., Ltd. and all the authentication of plant materials was identified by Dr. Jian Ni at Beijing University of Chinese Medicine. The voucher specimens (Voucher numbers: A. membranaceus (Fisch.) Bunge-2016-007; S. Miltiorrhiza Bunge-2016-008; L. japonica Thunb-2016-009; S. aestivalis Griseb-2016-010; A. fischeri Rchb-2016-011; G. uralensis Fisch2016-012) were submitted to Department of Chinese medicine teaching and Research, School of Traditional Chinese Medicine, Beijing University of Chinese Medicine (Wang et al., 2017). The previous team tested the chemical composition of QSG by 
Ultra-high performance liquid chromatography coupled with hybridion trap-time of flight mass spectrometry (UHPLCIT-TOF-MS), and the results showed that Glycyrrhizic acid, Formononetin, Dihydrotanshinone I, Cryptotanshinone, Tanshinone I, Tanshinone I, Glycyrrhetic acid, and Tanshinone IIA were the main component (Guo et al., 2016). The fingerprint of QSG was analyzed by HPLC and the typical chromatograms were shown in Supplementary Data 1. Fosinopril was provided by Sino-US Shanghai Squibb Pharmaceutical Co. Ltd. Country Medicine Accurate Character Number: H19980197.

\section{Animal Model of Heart Failure and Drug Administration}

Left anterior descending (LAD) coronary artery of rats was ligated to induce the HF model as described in our previous studies (Wang et al., 2013). Briefly, 60 SD rats underwent left thoracotomy between the third and fourth intercostal space. After exposure of heart tissues, the LAD was ligated with a sterile suture $1 \mathrm{~mm}$ below the left atrium in 50 rats. Rats in sham group only underwent thoracotomy and threading in the same position of the heart. Ten rats that underwent LAD ligation were randomly chosen for splenectomy surgery using previously published method (Schwarz and Hiserodt. 1990). Approximately $1 \mathrm{~cm}$ incision was made by midline laparotomy, hepatic hilum was clamped, spleen was removed, and the muscles and skin were sutured with silk suture.

The main causes of mortality in myocardial infarction rats were anesthesia, laryngeal edema, excessive blood loss, arrhythmia, lung injury, pneumothorax, etc (Degabriele et al., 2004). Within 1 day after surgery, 8 rats died ( 1 rat in the splenectomy group) and 52 survived. The survival rate was $86.7 \%$. 52 rats were randomly divided into five groups: 10 rats in the sham group, 11 rats in the model group, 9 rats in the splenectomy group, 11 rats in the QSG group, and 11 rats in the fosinopril group.

Rats in QSG group were treated with QSG at the dosage of $235.2 \mathrm{mg} / \mathrm{kg}$ per day and rats in fosinopril group were treated with fosinopril at the dosage of $4.67 \mathrm{mg} / \mathrm{kg}$ per day for $21 \mathrm{~d}$. All the drugs were gavaged with the amount of $1 \mathrm{ml} / 100 \mathrm{~g}$. Rats in the sham group, model group and splenectomy group were administrated with the same volume of water for $21 \mathrm{~d}$.

\section{Evaluation of Echocardiographic Parameters}

$21 \mathrm{~d}$ after treatment, rats inhaled anesthetic isoflurane and vevo 2100 was applied to observe the echocardiographic parameters of rats to assess the cardiac functions (Vevo TM 2100, Visual Sonics, Canada). Short axis was used to measure the length of left ventricular anterior wall; systole (LVAW;s), left ventricular anterior wall; diastole (LVAW;d), left ventricular internal diameter; diastole (LVID; d), left ventricular internal diameter; systole (LVID;s), left ventricular posterior wall; systole (LVPW;s), left ventricular posterior wall; diastole (LVPW;d). The values of ejection fraction (EF) and fractional shortening (FS) were calculated according to the following equation: $\mathrm{FS} \%=[(\mathrm{LVID} ; \mathrm{d}-\mathrm{LVID} ; \mathrm{s}) / \mathrm{LVID} ; \mathrm{d}] \times 100 \% ; \mathrm{EF} \%=[(\mathrm{LVEDV}-$ LVESV)/LVEDV] $\times 100 \%$.

\section{Histological Examination}

The heart and spleen tissues were fixed in $4 \%$ paraformaldehyde for $48 \mathrm{~h}$, and then embedded in paraffin and cut into $5 \mu \mathrm{m}$ sections. The spleen tissues were stained with hematoxylin-eosin (HE) staining to assess the basic structure. The heart tissues were stained with Masson staining and Sirius red staining to assess the extent of inflammatory infiltration and myocardial fibrosis.

\section{Measurement of angiotensin II in the serum by radioimmunoassay}

The level of angiotensin II was quantified directly from prepared plasma using radioimmunoassay (RIA) kits (Beijing Sino-UK institute of biological technology, Beijing, China) according to protocol of the manufacturer.

\section{Measurement of Indicators by Immunohistochemistry}

The heart sections were deparaffinized in xylene, rehydrated in alcohol gradient and then rinsed in PBS. The samples were incubated with primary antibody (Anti-CD31, GB12063, Servicebio, China) for $12 \mathrm{~h}$ at $4^{\circ} \mathrm{C}$ after being blocked with $0.3 \%$ hydrogen peroxide, avidin biotin blocking reagent, and biotin blocking reagent for $15 \mathrm{~min}$. The slides were then incubated with the biotinylated secondary antibody for $2 \mathrm{~h}$ and the stained with hematoxylin for $30 \mathrm{~s}$. The slides were then dehydrated by graded ethanol and xylene and fixed with resin glue. The positive area was specifically stained brown-yellow.

\section{Measurement of Indicators by Immunofluorescence}

The sections were sequentially dewaxed in xylene I and II, rehydrated in an alcohol gradient, and then placed in a citrate antigen retrieval buffer ( $\mathrm{pH}$ 8.0) for antigen retrieval. Normal serum was added to the slides and blocked at room temperature for $30 \mathrm{~min}$. After blocking, sections were incubated overnight with anti-CD68 (ab201340, Abcam, United States), anti-CD86 (ab53004, Abcam, United States), anti-CD163 (ab182422, Abcam, United States) primary antibody at $4^{\circ} \mathrm{C}$ and with fluorescent secondary antibody for $1 \mathrm{~h}$ at $37^{\circ} \mathrm{C}$. The sections were further stained with DAPI staining for $5 \mathrm{~min}$ in the dark. Finally, the sections were sealed on cover slips and fluorescence images were captured using fluorescence microscope.

\section{Measurement of Indicators by Western Blot}

Tissue protein of heart and spleen was lysed by the RIPA lysate (50:1) and tissue disrupter and quantified by the BCA method. The quantified homogenate was added to loading buffer, and the mixture was boiled and denatured at $99^{\circ} \mathrm{C}$. Each lane was loaded with $10 \mathrm{ul}$ of proteins. After electrophoresis at $100 \mathrm{~V}$ for 1-1.5 h, proteins were transferred to PVDF membranes at $300 \mathrm{~mA}$ for $1-1.5 \mathrm{~h}$. Afterwards, the membrane blocked with $5 \%$ skim milk for $1-1.5 \mathrm{~h}$ at room temperature, incubated on a shaker, and washed with TBS-T. Western blot analysis was conducted using anti-AT1 (ab18801; Abcam, United States), 
anti-MCP-1 (ab25124, Abcam, United States), anti-CCR2 (PAI-27409), anti-TGF- $\beta 1$ (3711s, Cell Signaling Technology, Germany), anti-Smad3 (ab28379, Abcam, United States), antiMMP2 (ab86607, Abcam, United States), anti-Col III (ab7778, Abcam, United States), anti-VEGF (ab10972, Abcam, United States), anti-CD31 (ab24590, Abcam, United States) and antiGAPDH (ab8245, Abcam, United States) at $4^{\circ} \mathrm{C}$ overnight. After incubation with the appropriate secondary antibodies at $37^{\circ} \mathrm{C}$ for $2 \mathrm{~h}$ at room temperature, the membrane treated with ECL for $1 \mathrm{~min}$ at room temperature. The final expression of each protein was normalized by GAPDH and grayscale analysis was performed using Image J software.

\section{Statistical Analysis}

All data were expressed as mean \pm standard deviation (SD). Oneway analysis of variance (ANOVA) and Dunnett's test were used to compare differences among multiple groups. The values of $P<$ 0.05 were considered as statistically significant. All analyses were performed using SPSS 17.0 or GraphPad Prism 7.

\section{RESULTS}

\section{QSG Inhibited the Release of Monocytes From the Spleen}

Heart failure results in increased motility of monocytes in the spleen (Swirski FK et al., 2009). First, we assessed the number of monocyte in the spleen on $21 \mathrm{~d}$ after LAD ligation. The subcapsular region is the site where monocytes are stored in the spleen. HE staining showed that the number of monocytes in the marginal zone of the spleen was decreased after LAD ligation compared with the sham group, indicating that after LAD ligation, spleen released a large number of monocytes. Second, the effect of QSG on the release of monocytes from spleen was investigated. QSG could significantly increase the number of monocytes in red pulp under the capsule and marginal zone of the spleen as compared with those in the model group (Figure 1A). Fosinopril, an angiotensin-converting enzyme inhibitor (ACEI), was applied as the positive control drug. Circulatory level of angiotensin II in model group was increased compared with sham group. Consistently, levels of AT1 and MCP-1 in the spleen were also up-regulated in the model group. Treatment of QSG and fosinopril could significantly down-regulate the levels of AngII, AT1 and MCP-1, indicating that QSG could suppress the release of monocytes from the spleen via inhibiting Ang II/ AT1 signaling pathway (Figures 1B, C).

\section{QSG Inhibited Recruitment of Monocytes and M1 Macrophages-Induced Activation of Transforming Growth Factor (TGF)-B1/ Smad3 in the Border Zone of Infarcted Heart}

The effect of QSG on the recruitment of monocytes to infarcted heart tissues was further examined. Immunofluorescence results showed that the number of M1 macrophages (CD68 + CD86 positive cells) increased significantly in the model group relative to the sham group. QSG could reduce the number of M1 macrophages in the border zone of infarcted heart as compared with the model group. Splenectomy was applied to investigate whether the increased macrophages in heart were originated from the spleen. After splenectomy, the number of M1 macrophages
A
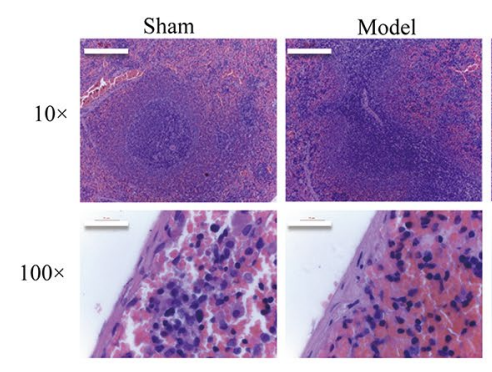

C

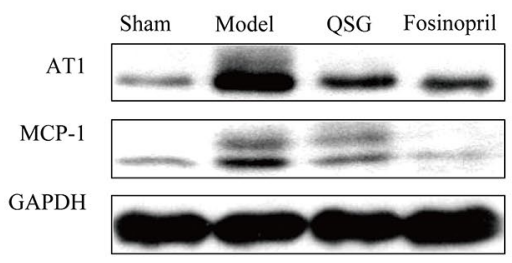

QSG
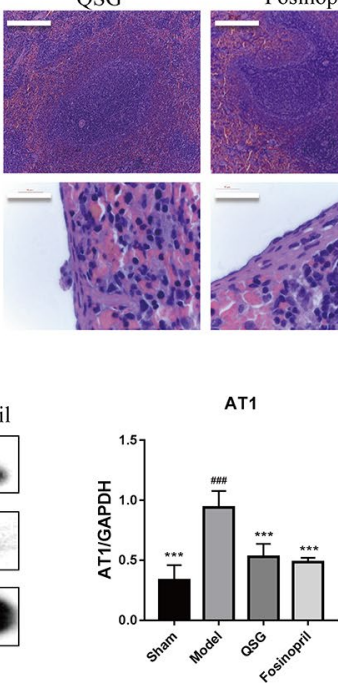

Fosinopril
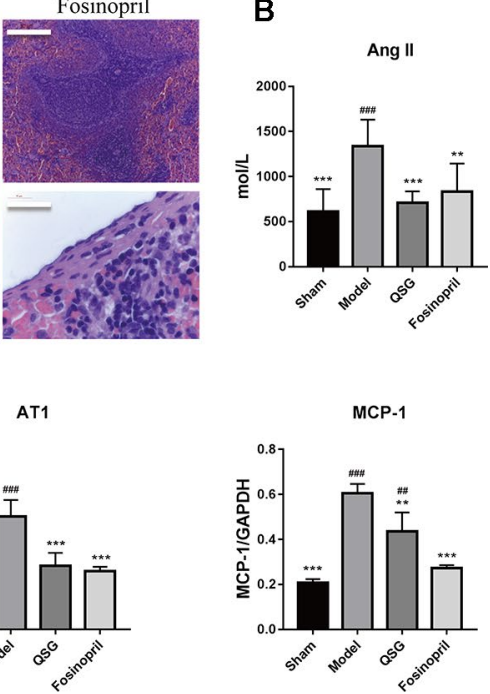

FIGURE 1 | QSG inhibited the release of monocytes from the spleen. (A) HE staining showed that monocytes in the marginal zone of the spleen were released in the model group, and QSG could exert the protective effect by increasing the number of monocytes in red pulp under the capsule and marginal zone of the spleen. (B). QSG significantly decreased the circulatory level of angiotensin II. (C) Western blot showed that QSG reduced the expressions of AT1 and MCP-1 in splenic tissues. All data were presented as means \pm SD from independent experiments performed in triplicate. ${ }^{\# \#} P<0.01,{ }^{\# \# \#} P<0.001$ vs the sham group; ${ }^{\star \star} P<0.01$, ${ }^{* \star *} P<0.001$ vs the model group. $\mathrm{N}=3$ per group. 
was reduced significantly (Figure 2A). Splenectomy could also significantly alleviate inflammation and fibrosis in the heart tissues, demonstrating that splenic monocytes played important role in myocardial remodeling (Figure 2B). Since AT1 receptoractivated MCP-1/CCR2 interaction is critically involved in the recruitment of monocytes to heart tissues (França et al., 2017), the effects of QSG on the expressions of AT1, MCP-1 and CCR2 were then examined. Results showed that the levels of AT1, MCP-1 and CCR2 were significantly increased in the model group, as compared with the sham group. After treatment with QSG, expressions of AT1, MCP-1 and CCR2 were down-regulated, indicating that QSG could inhibit recruitment of monocytes through MCP-1/CCR2 interactions. Splenectomy and fosinopril had similar effects as QSG treatment (Figure 2C).

When monocytes are recruited to heart tissues, they could differentiate into M1 macrophages, which are able to stimulate production of TGF- $\beta 1$ by fibroblasts, activate Smad 3 and induce production of collagens and MMPs, thereby promoting fibrosis and myocardial remodeling (Shivshankar et al., 2014). Western blot showed that QSG could down-regulate expressions of TGF$\beta 1$ and Smad 3 in the border zone of myocardial infarction. Levels of collagen III and matrix metallopeptidase 2 (MMP2) were
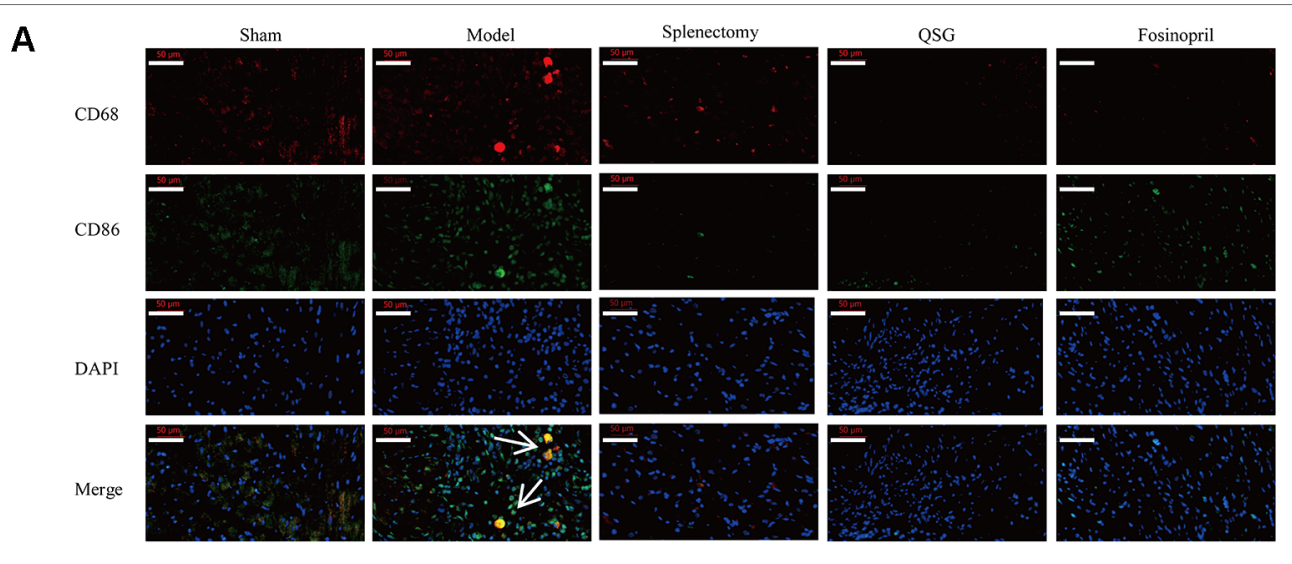

B
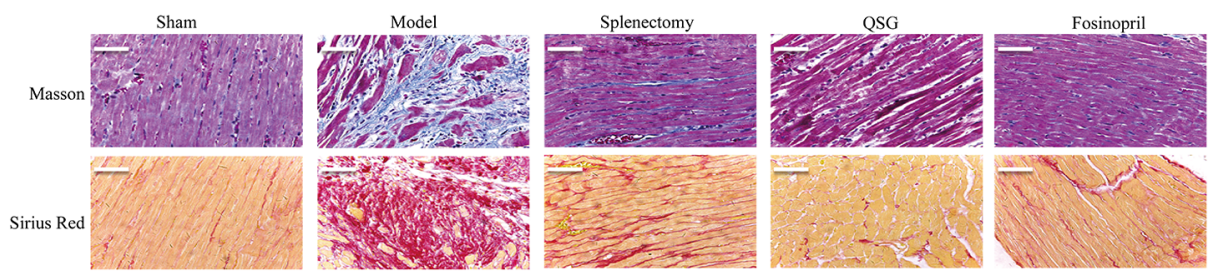

C
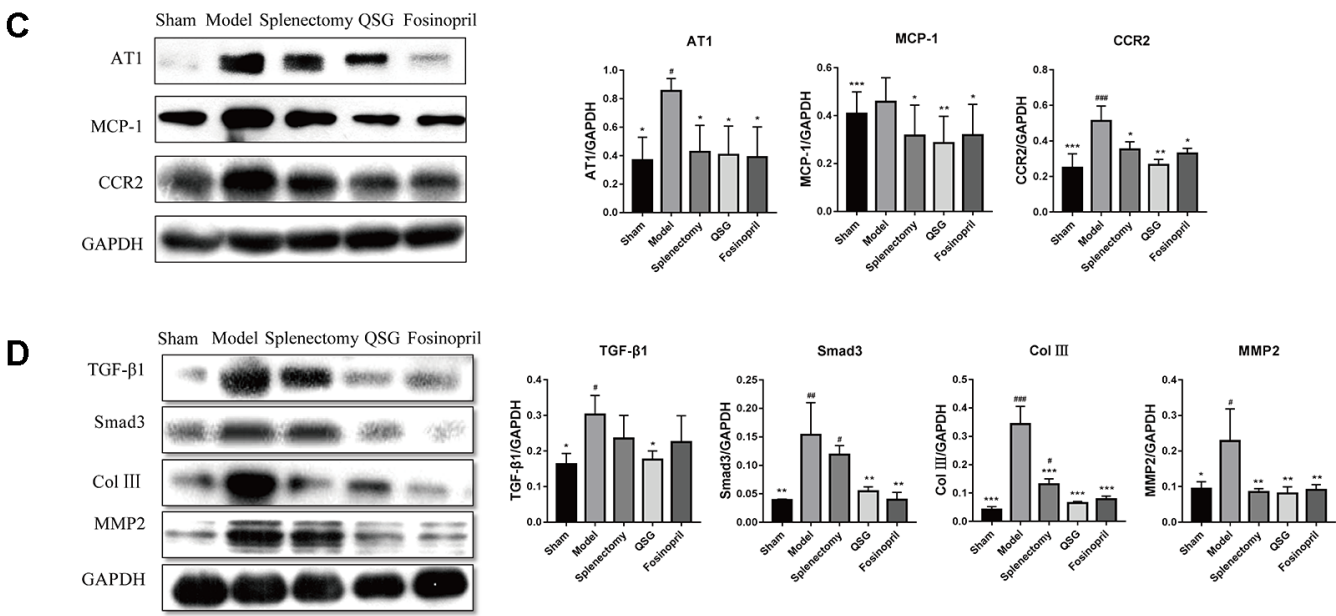

FIGURE 2 | QSG inhibited activation of TGF- $\beta 1 /$ Smad3 through reducing the recruitment of M1 macrophages in myocardial tissue. (A) IF staining showed that splenectomy reduced the recruitment of M1 macrophages in myocardial tissue; QSG treatment decreased the recruitment of M1 macrophages. (B) Masson and Sirius Red staining showed that QSG inhibited cardiac fibrosis. (C) Western blot showed that QSG down-regulated the expressions of AT1, MCP-1 and CCR2 in myocardial tissue compared with that in the model group. (D) Western blot showed that QSG decreased the expressions of TGF- $\beta 1$, Smad3, collagen III and MMP2 in cardiac tissue. All data were presented as means \pm SD from independent experiments performed in triplicate. $\# P<0.05, \# P<0.01, \# \# P<0.001$ vs the sham group; ${ }^{*} P<0.05,{ }^{\star \star} P<0.01,{ }^{\star \star \star} P<0.001$ vs the model group. $\mathrm{N}=3$ per group. 
also reduced by QSG treatment. Splenectomy and fosinopril had similar effects (Figure 2D). These results suggested that QSG could ameliorate myocardial remodeling by inhibiting macrophage-activated TGF- $\beta 1 / \mathrm{Smad} 3$ pathway.

\section{QSG Induced Differentiation of M2 Macrophages and Promoted Angiogenesis in Myocardial Tissue}

In contrast to M1 macrophages, M2 macrophages are involved in inflammation suppression, tissue repair and angiogenesis (Ribeiro et al., 2018). Therefore, the effect of QSG on differentiation of M2 macrophages was further investigated. Immunofluorescence staining (labeled by CD86 and CD163) showed that the number of M2 macrophages in QSG group was increased compared with model group. In splenectomy group, there were no detectable M2 macrophages (Figure 3A). Immunohistochemical staining of CD31 showed that the number of microvessels was significantly up-regulated after treatment with QSG. However, the effect of splenectomy group was less significant than of QSG group (Figure 3B). Western blot showed that compared with the sham group, the expression levels of VEGF and CD31 were significantly down-regulated in the model group. After treatments with QSG, the expression levels of VEGF and CD31 were increased (Figure 3C), demonstrating that QSG might promote angiogenesis through M2 macrophages. Fosinopril had similar effect with QSG. Echocardiography showed that compared with the sham group, left ventricular ejection fraction (EF) and fractional shortening (FS) of the model group were significantly decreased $(P<0.001)$. QSG increased EF by $56 \%$, demonstrating that QSG could improve the left ventricular contractility. Splenectomy and fosinopril could also improve heart function (Figure 4).

\section{DISCUSSION}

Myocardial remodeling occurs in a variety of cardiovascular diseases and can be caused by inflammatory response, which
A

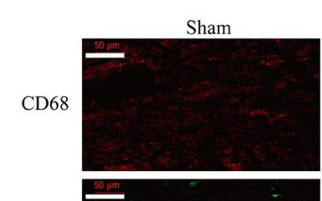

$\mathrm{CD} 163$
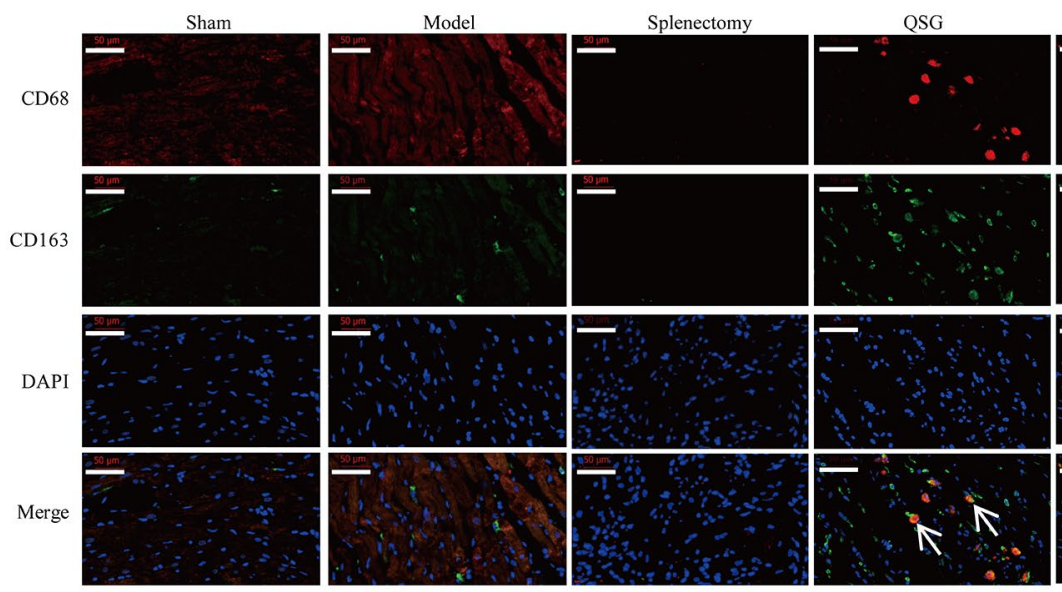

B
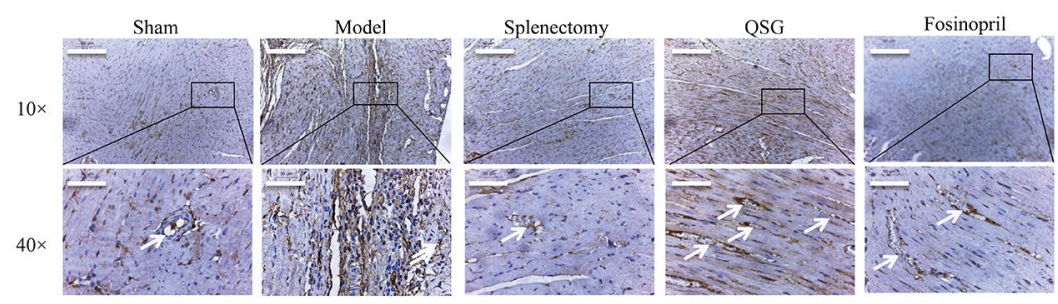

C

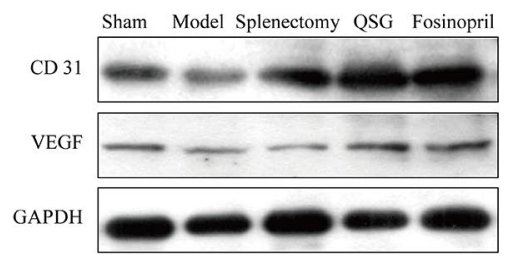

CD31

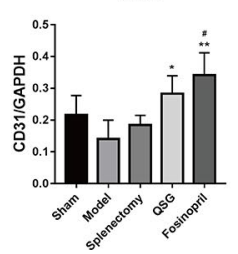

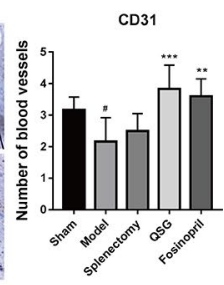
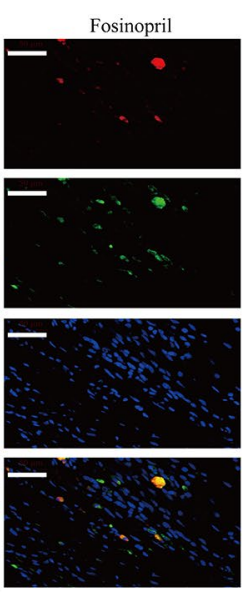

CD31

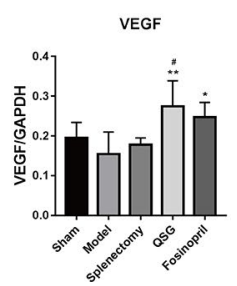

FIGURE 3 | QSG promoted angiogenesis through inducing the number of M2 macrophages in myocardial tissue. (A) IF staining showed that QSG treatment up-regulated the M2 macrophages. After splenectomy, the number of M2 macrophages remained unchanged relative to the model group. (B) CD31 staining showed that QSG could promote angiogenesis. (C) Western blot showed that QSG increased the expressions of CD31 and VEGF in myocardial tissue compared with model group. All data were presented as means \pm SD from independent experiments performed in triplicate. ${ }^{\sharp} P<0.05$ vs the sham group; ${ }^{\star} P<0.05$, ${ }^{\star \star} P<0.01,{ }^{\star \star \star} P<0.001$ vs the model group. $\mathrm{N}=3$ per group. 
A

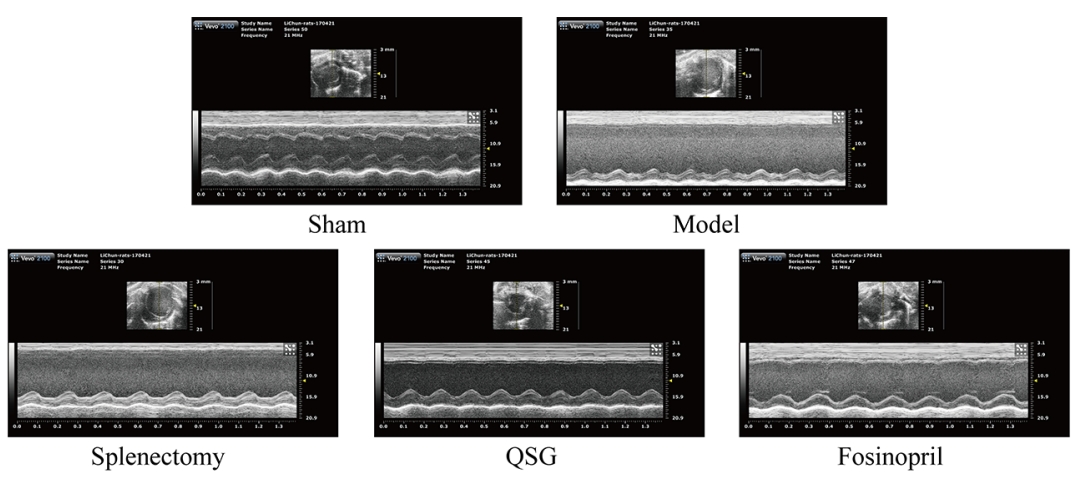

B

LVAW;d

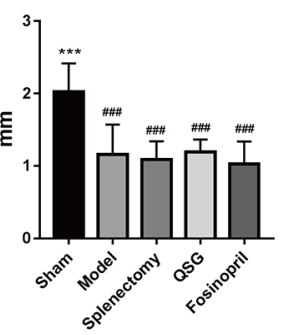

LVID;d

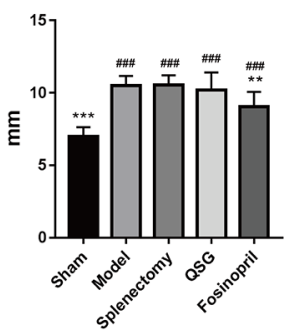

LVAW;s

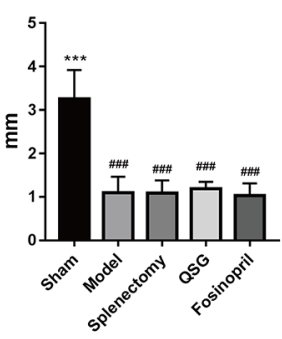

LVID;s

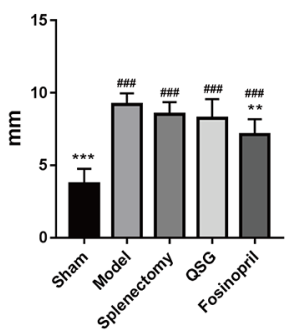

LVPW;d

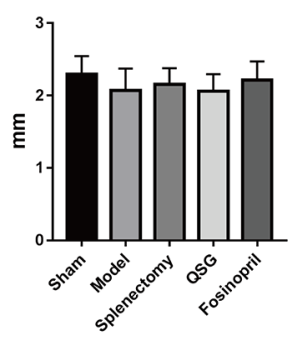

LVEF

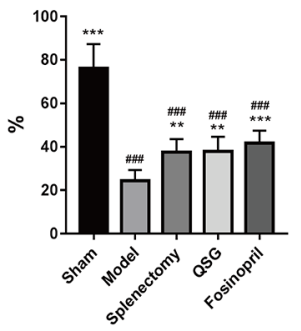

LVPW;s

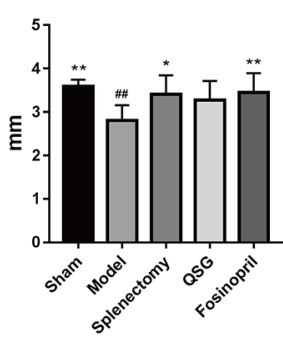

LVFS

FIGURE 4 | QSG improves cardiac function by echocardiography. (A) Representative images of echocardiography exhibiting the changes in cardiac function in each group. (B) Echocardiography results showed that QSG and splenectomy improved left ventricular ejection fraction, and cardiac function of $\mathrm{HF}$ rats. \#\# $P$. 0.01 , ${ }^{\# \# \# P} 0.001$ vs the sham group; ${ }^{\star} P<0.05,{ }^{* \star} P<0.01,{ }^{\star \star \star} P<0.001$ vs the model group. $\mathrm{N}=7$ per group.

is closely related to infiltration of macrophages (Frantz and Nahrendorf, 2014). Macrophage is an important cell population in healthy heart tissues. Macrophages have multiple phenotypes and functions. It is speculated that macrophages guard against infection and regulate matrix turnover under steady state, although the source of macrophages in healthy heart remains unclear (Davies and Taylor. 2015). Under inflammatory conditions, such as acute infarction, circulatory monocytes will be recruited to heart tissues and differentiate into different types of macrophages. These macrophages play critical roles in inflammation, fibrosis, angiogenesis and myocardial remodeling. Therefore, regulating the recruitment of monocytes and differentiation of macrophages is considered to be promising target for managing myocardial remodeling. In this study, we explored the effect of traditional Chinese medicine QSG on monocytes/macrophages in infarct heart and spleen tissues and investigated the mechanism by which QSG inhibited fibrosis and myocardial remodeling.
A rat model of LAD ligation was induced in this study. LAD ligation led to myocardial infarction, remodeling and eventual heart failure. We firstly explored the source of macrophages in the border zone of infarction site. In addition to bone marrow, the spleen is a reservoir of monocytes and may supply large amount of monocytes that can be recruited to heart tissues under inflammatory conditions. Studies have shown that $40-70 \%$ of the macrophages in myocardial infarct site are recruited from spleen (Okizaki et al., 2015). Some experiments have demonstrated that removal of the spleen could inhibit the release of splenic monocytes and protect heart function (Pinto et al., 2012). Therefore, we performed splenectomy to explore the role of splenic monocytes under myocardial infarction conditions. It was shown that $21 \mathrm{~d}$ after LAD ligation, there were infiltrations of M1 macrophages (inflammatory type) in heart tissues. Myocardial fibrosis and compromised heart function were also observed. Furthermore, the number of monocytes in the spleen was significantly reduced in the model group. After removal of 
spleen, the number of M1 macrophages in myocardial tissue was significantly reduced and fibrosis was attenuated. Heart function was also improved by splenectomy. These results demonstrate that spleen is an important source of M1 macrophages after occurrence of myocardial infarction. Inhibition of release of splenic monocytes could attenuate fibrosis and protect against heart failure.

Furthermore, release of splenic monocytes is dependent on activation of renin-angiotensin-aldosterone system (RAAS). Angiotensin II activates release of splenic monocytes through AT1 receptor, and ACE inhibitor can attenuate release of monocytes from spleen (Leuschner et al.2010). Our previous studies have indicated that QSG has anti-inflammatory effects (Wang et al, 2015). Therefore, the effect of QSG on release of splenic monocytes was investigated in current study. Impressively, we found that QSG treatment could inhibit release of monocytes from spleen in rats with LAD ligation, and circulatory level of angiotensin II was also reduced accompanied by the suppression of AT1 and MCP-1. These data suggest that QSG exerts antiinflammatory effect by suppressing release of splenic monocytes through the AT1-MCP-1 pathway in spleen. Under myocardial infarction conditions, the monocytes released by spleen will be recruited to heart through MCP-1/CCR2 interaction. In heart tissues, monocytes will be differentiated into inflammatory M1 macrophages and alternative activated M2 macrophages (Moore and Tabas. 2011). M1 macrophages contribute to myocardial fibrosis by stimulating production of TGF- $\beta 1$, which contributes to tissue fibrosis by stimulating Smads signaling and releasing extracellular matrix from myocardial myofibroblasts (Zhang et al., 2016). In our study, we found that QSG could reduce the number of M1 macrophages in the border zone of myocardial infarction and inhibit deposition of collagens. The expressions of AT1, MCP-1 and CCR2 in heart tissues were reduced accordingly. Furthermore, the levels of TGF- $\beta 1$ and Smad 3 were also suppressed. ACE inhibitor had similar effect as those of QSG. These data indicate that QSG may inhibit monocyte recruitment via MCP-1/CCR2 interaction and suppress M1 macrophageinduced fibrosis via TGF- $\beta 1 / \mathrm{Smad} 3$ pathway.

In addition, studies have shown that M1 macrophages are inflammatory whereas M2 macrophages participate in tissue repair, angiogenesis and resolution of inflammation (Liao et al., 2018). Promoting differentiation of monocytes into M2 macrophages might be beneficial for heart function (Singla et al, 2017). In this study, we observed that the number of M2 macrophages was up-regulated by QSG treatment. Intriguingly, there were no M2 macrophages observed in heart tissues in rats with splenectomy, as the reservoirs of monocytes, the spleen had been removed. Previous studies showed that M2 macrophages could promote angiogenesis by excreting VEGF (Oka et al., 2014). We found that the number of microvessels was significantly up-regulated after QSG treatment and the expressions of CD31 and VEGF were also increased by QSG. These results demonstrate that QSG could exert angiogenic effects through up-regulating differentiation of M2 macrophages.

\section{\begin{tabular}{l|l|l|l|l|l|l|l|l|l|l|l|l}
0 & 0 & 0 & 0 & 0 & 0 & 0 & 0 & 0 & 0 & 0 & 0 & 0
\end{tabular}}

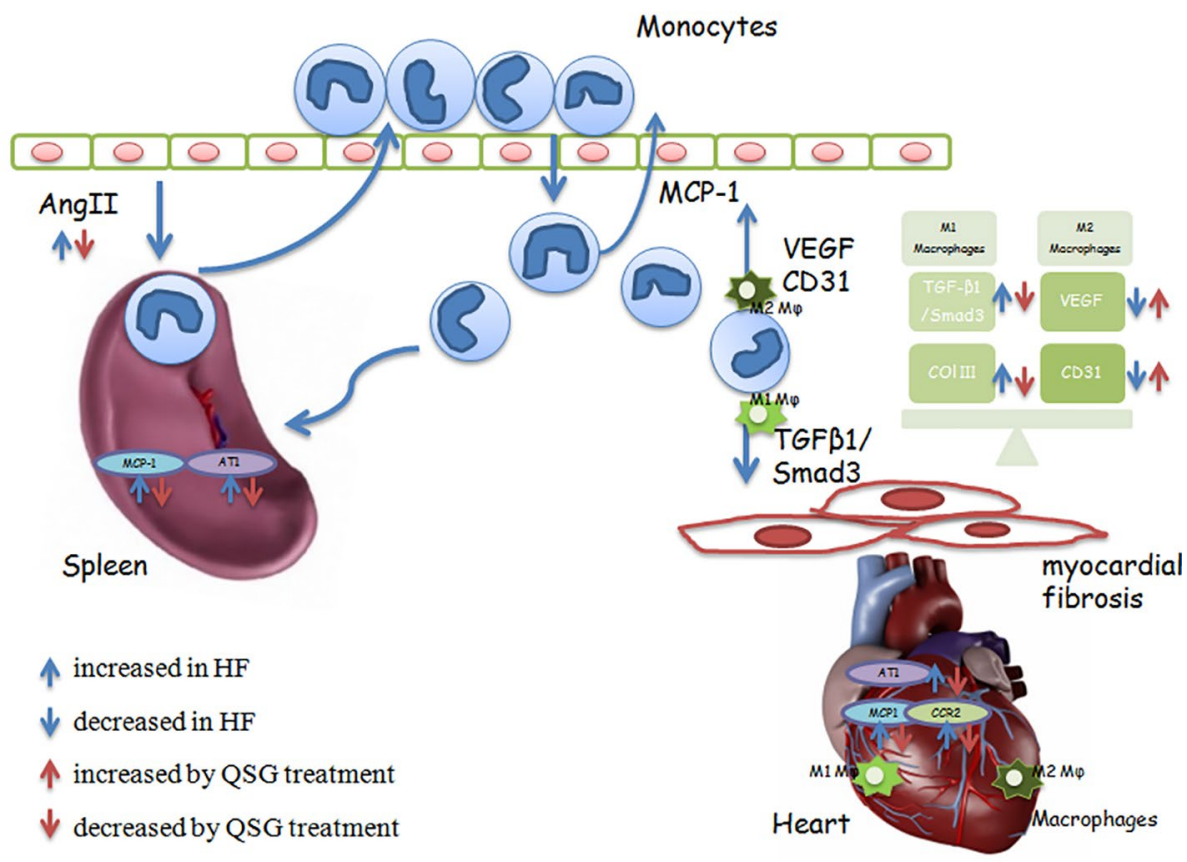

FIGURE 5 | QSG suppresses the release of spleen monocytes and recruitment to heart tissues through the splenic Ang II/AT1-cardiac MCP-1/CCR2 pathway to inhibit MR. 
In conclusion, QSG attenuated myocardial fibrosis through restoring the unbalance of M1 and M2 macrophages in heart tissues. Monocytes released by spleen could be recruited to heart tissues while QSG could suppress splenic release of monocytes. QSG could inhibit M1 macrophages-activated TGF- $\beta 1 / \mathrm{Smad} 3$ pathway and promote M2 macrophage-dependent angiogenesis (Figure 5). This study provides evidence that compounds of Chinese medicine have synergistic effect on cardiac and splenic organs. Moreover, restoring the balance of M1 and M2 macrophages can be a promising strategy for the management of myocardial remodeling.

\section{DATA AVAILABILITY STATEMENT}

All datasets generated for this study are included in the article/ Supplementary Material.

\section{ETHICS STATEMENT}

All animal experimental protocols were approved by the Ethics Committee of Beijing University of Chinese Medicine and conformed to the Guide for the Care and Use of Laboratory Animals published by the U.S. National Institute of Health (NIH Publication No. 85-23, revised 1996).

\section{REFERENCES}

Davies, L. C., and Taylor, P. R. (2015). Tissue-resident macrophages: then and now. Immunol. 144 (4), 541-548. doi: 10.1111/imm.12451

Degabriele, N. M., Griesenbach, U., Sato, K., Post, M. J., Zhu, J., Williams, J., et al. (2004). Critical appraisal of the mouse model of myocardial infarction. Exp. Physiol. 89 (4), 497-505. doi: 10.1113/expphysiol.2004.027276

França, C. N., Izar, M. C. O., Hortêncio, M. N. S., Do Amaral, J. B., Ferreira, C. E. S., Tuleta, I. D., et al. (2017). Monocyte Subtypes and the CCR2 Chemokine Receptor in Cardiovascular Disease. Clin. Sci. (Lond) 131, 1215-1224. doi: 10.1042/CS20170009

Frantz, S., and Nahrendorf, M. (2014). Cardiac Macrophages and Their Role in Ischaemic Heart Disease. Cardiovasc. Res. 102 (2), 240-248. doi: 10.1093/Cvr/ Cvu025

Guo, S., Li, P., Fu, B., Chuo, W., Gao, K., Zhang, W., et al. (2016). Systems-Biology Dissection of Mechanisms and Chemical Basis of Herbal Formula in Treating Chronic Myocardial Ischemia. Pharmacol. Res. 114, 196-208. doi: 10.1016/J. Phrs.2016.10.026

Halade, G. V., Norris, P. C., Kain, V., Serhan, C. N., and Ingle, K. A. (2018). Splenic Leukocytes Define the Resolution of Inflammation in Heart Failure. Sci. Signaling 11 (520), Eaao1818. doi: 10.1126/Scisignal.Aao1818

Honold, L., and Nahrendorf, M. (2018). Resident and Monocyte-Derived Macrophages in Cardiova-scular Disease. Circ. Res. 122 (1), 113-127. doi: 10.1161/CIRCRESAHA.117.311071

Hu, Y., Jiang, S., Lu, S., Xu, R., Huang, Y., Zhao, Z., et al. (2017). Echocardiography and Electrocardiography Variables Correlate With the New York Heart Association classification: An Observational Study of Ischemic Cardiomyopathy Patients. Med 96 (26), e7071. doi: 10.1097/MD.0000000000007071

Hulsmans, M., Sager, H. B., Roh, J. D., Valero-Muñoz, M., Houstis, N. E., Iwamoto, Y., et al. (2018). Cardiac macrophages promote diastolic dysfunction. J. Exp. Med. 215 (2), j423-j440. doi: 10.1084/jem.20171274

Jablonski, K. A., Amici, S. A., Webb, L. M., Ruiz-Rosado Jde, D., Popovich, P. G., Partida-Sanchez, S., et al. (2015). Novel Markers to Delineate Murine M1 and M2 Macrophages. PloS One 10 (12), E0145342. doi: 10.1371/Journal. Pone.0145342

\section{AUTHOR CONTRIBUTIONS}

CL, YWa and YL designed the study. WL and QiyW did majority of experimental work and interpretation throughout the experiments. XS, HH and QixW analyzed the data. YWu operated cardiac ultrasound. All authors read and approved the final manuscript.

\section{FUNDING}

This work was supported by the Grants from the National Natural Science Foundation of China (Nos. 81673802, 81530100, 81673712, and 81822049), Fok Ying Tung Education Foundation (No. 151044), Beijing Nova program (No. Z171100001117028), Talent Young Scientist of China Association for Science and Technology (No. CACM-2017-QNRC2-C13, No. CACM-2018QNRC2-C07), the Fundamental Research Funds for the Central Universities (2017-JYB-JS-020).

\section{SUPPLEMENTARY MATERIAL}

The Supplementary Material for this article can be found online at: https://www.frontiersin.org/articles/10.3389/fphar.2019.01399/ full\#supplementary-material

Leuschner, F., Panizzi, P., Chico-Calero, L., Lee, W. W., Ueno, T., Cortez-Retamozo, V. et al. (2010). ACE Inhibition Prevents the Release of Monocytes From Their Splenic Reservoir in Mice With Myocardial Infarction. Circ. Res. 107 (11), 1364-1373. doi: 10.1161/CIRCRESAHA.110.227454

Li, C., Wang, Y., Qiu, Q., Shi, T., Wu, Y., Han, J., et al. (2014). Qishenyiqi Protects Ligation-Induced Left Ventricular Remodeling by Attenuating Inflammation and Fibrosis via STAT3 and NF-кB Signaling Pathway. PloS One 9 (8), e104255. doi: 10.1371/journal.pone.0104255

Li, C., Wang, J., Wang, Q., Zhang, Y., Zhang, N., Lu, L., et al. (2016). Qishen granules inhibit myocardial inflammation injury through regulating arachidonic acid metabolism. Sci. Rep. 6, 36949. doi: 10.1038/srep36949

Liao, X., Shen, Y., Zhang, R., Sugi, K., Vasudevan, N. T., Alaiti, M. A., et al. (2018). Distinct Roles of Resident and Nonresident Macrophages in Nonischemic Cardiomyopathy. Proc. Natl. Acad. Sci. 115 (20), E4661-E4669. doi: 10.1073/ Pnas. 1720065115

Liu, Z., Guo, F., Wang, Y., Li, C., Zhang, X., Li, H., et al. (2016). BATMAN-TCM: a Bioinformatics Analysis Tool for Molecular mechANism of Traditional Chinese Medicine. Sci. Rep 2016 6, 21146. doi: 10.1038/srep21146

Momtazi-Borojeni, A. A., Abdollahi, E., Nikfar, B., Chaichian, S., Ekhlasi-Hundrieser, M. et al. (2019). Curcumin as a potential modulator of M1 and M2 macrophages: new insights in atherosclerosis therapy. Heart Failure Rev. 24 (3), 399-409. doi: 10.1007/s10741-018-09764-z

Moore, K. J., and Tabas, 1. (2011). Macrophages in the pathogenesis of atherosclerosis. Cell. 145 (3), 341-355. doi: 10.1016/j.cell.2011.04.005

Oka, T., Akazawa, H., and Naito, A. T. (2014). Angiogenesis and Cardiac Hypertrophy: Maintenance of Cardiac Function and Causative Roles in Heart Failure. Circ. Res. 114 (3), 565-571. doi: 10.1161/CIRCRESAHA.114. 300507

Okizaki, S., Ito, Y., Hosono, K., Oba, K., Ohkubo, H., Amano, H., et al. (2015). Suppressed recruitment of alternatively activated macrophages reduces TGF$\beta 1$ and impairs wound healing in streptozotocin-induced diabetic mice. Biomed Pharmacother 70, 317-325. doi: 10.1016/j.biopha.2014.10.020

Partha D. Nahrendorf, M. (2015). Monocytes in Myocardial Infarction. Arterioscler. Thromb. Vasc. Biol. 35 (5), 1066-1070. doi: 10.1161/ATVBAHA. 114.304652 
Pinto, A. R., Paolicelli, R., Salimova, E., Gospocic, J., Slonimsky, E., Bilbao-Cortes, D. et al. (2012). An Abundant Tissue Macrophage Population in the Adult Murine Heart with a distinct alternatively-activated macrophage profile. PloS One 7 (5), e36814. doi: 10.1371/journal.pone.0036814

Pryds, K., Rahbek Schmidt, M., Bjerre, M., Thiel, S., Refsgaard, J., Bøtker, H. E., et al. (2019). Effect of long-term remote ischemic conditioning on inflammation and cardiac remodeling. Scand Cardiovasc. J. 5, 1-9. doi: 10.1080/14017431.2019.1622770

Ribeiro, K. C., Campelo, R. P., Rodrigues, D. D. R. F., Mattos, E. C., Brandão, I. T., da Silva, C. L., et al. (2018). Immunization with plasmids encoding M2 acetylcholine muscarinic receptor epitopes impairs cardiac function in mice and induces autophagy in the myocardium. Autoimmunity 51 (5), 245-257. doi: 10.1080/08916934.2018.1514389

Robbins, C. S., and Swirski, F. K. (2010). The multiple roles of monocyte subsets in steady state and inflammation. Cell. Mol. Life Sci. 67 (16), 2685-2693. doi: 10.1007/s00018-010-0375-x

Sager, H. B., Kessler, T., and Schunkert, H. (2017). Monocytes and macrophages in cardiac injury and repair. J. Thoracic Dis. 9 (Suppl 1), S30-S35. doi: 10.21037/ jtd.2016.11.17

Schwarz, R. E., and Hiserodt, J. C. (1990). Effects of splenectomy on the development of tumor-specific immunity[J]. J. Surg. Res. 48 (5), 448-453. doi: 10.1016/0022-4804(90)90011-P

Shivshankar, P., Halade, G. V., Calhoun, C., Escobar, G. P., Mehr, A. J., Jimenez, F., et al. (2014). Caveolin-1 deletion exacerbates cardiac interstitial fibrosis by promoting M2 macrophage activation in mice after myocardial infarction. J. Mol. Cell. Cardiol. 76, 84-93. doi: 10.1016/j.yjmcc.2014.07.020

Singla, D. K., Wang., Jing, and Reetu, S. (2017). Primary human monocytes differentiate into M2 macrophages and involve Notch-1 pathway. Can. J. Physiol. pharamacology 95 (3), 288-294. doi: 10.1139/cjpp-2016-0319

Swirski, FK1, Nahrendorf, M., Etzrodt, M., Wildgruber, M., Cortez-Retamozo, V., Panizzi, P., et al. (2009). Identification of splenic reservoir monocytes and their deployment to inflammatory sites. Sci 325 (5940), 612-616. doi: 10.1126/science.1175202

Wang, J., Lu, L., Wang, Y., Wu, Y., Han, J., Wang, W., et al. (2015). Qishenyiqi Dropping Pill attenuates myocardial fibrosis in rats by inhibiting
RAAS-mediated arachidonic acid inflammation. J. ethnopharmacol 176, 375384. doi: 10.1016/j.jep.2015.11.023

Wang, Y., Lin, W., Li, C., Singhai, S., Jain, G., Zhu, R., et al. (2017). Multipronged Therapeutic Effects of Chinese Herbal Medicine Qishenyiqi in the Treatment of Acute Myocardial Infarction. Front. In Pharmacol. 2; 8, 98. doi: 10.3389/ fphar.2017.00098

Wang, Y., Li, C., Ouyang, Y., Shi, T., Yang, X., Yu, J., et al. (2013). QSYQ attenuates oxidative stress and apoptosis induced heart remodeling rats through different subtypes of NADPH-oxidase. Evid. Based Complement Alternat. Med. 2013, 824960. doi: 10.1155/2013/824960

Wang, Y., Li, C., Ouyang, Y., Yu, J., Guo, S., Liu, Z., et al. (2019). Cardioprotective Effects of Qishenyiqi Mediated by Angiotensin II Type 1 Receptor Blockade and Enhancing Angiotensin-Converting Enzyme 2. Evidence-Based Complement Altern Med. 2019, 9791436. doi: 10.1155/2019/9791436

Wu, W. K., Llewellyn, O. P., Bates, D. O., Nicholson, L. B., and Dick, A. D. (2010). IL-10 regulation of macrophage VEGF production is dependent on macrophage polarisation and hypoxia. Immunobiol 215 (9-10), 796-803. doi: 10.1016/j.imbio.2010.05.025

Zhang, F., Dang, Y., Li, Y., Hao, Q., Li, R., Qi, X., et al. (2016). Cardiac Contractility Modulation Attenuate Myocardial Fibrosis by Inhibiting TGF- $\beta 1 / \mathrm{Smad} 3$ Signaling Pathway in a Rabbit Model of Chronic Heart Failure. Cell. Physiol. Biochem. 39 (1), 294-302. doi: 10.1159/000445624

Conflict of Interest: The authors declare that the research was conducted in the absence of any commercial or financial relationships that could be construed as a potential conflict of interest.

Copyright (c) 2019 Lu, Wang, Sun, He, Wang, Wu, Liu, Wang and Li. This is an open-access article distributed under the terms of the Creative Commons Attribution License (CC BY). The use, distribution or reproduction in other forums is permitted, provided the original author(s) and the copyright owner(s) are credited and that the original publication in this journal is cited, in accordance with accepted academic practice. No use, distribution or reproduction is permitted which does not comply with these terms. 